\title{
SEMIGROUPS WITH ATOMISTIC CONGRUENCE LATTICES
}

\author{
KARL AUINGER
}

(Received 24 January 1990)

Communicated by P. G. Trotter

\begin{abstract}
In this note a characterization of semigroups with atomistic congruence lattices, given for weakly reductive semigroups, is generalized to arbitrary semigroups. Also, it is shown that there is a complete congruence on the congruence lattice of such a semigroup that decomposes it into a disjoint union of intervals of the partition lattice.
\end{abstract}

1991 Mathematics subject classification (Amer. Math. Soc.) 20 M 10, 08 A 30.

\section{Introduction and preliminaries}

A lattice $L$ is atomistic if each element of $L$ is the supremum of the atoms it contains. In [2] the author characterized weakly reductive semigroups whose congruence lattices are atomistic. These semigroups can be constructed by means of a locally finite tree $X, 0$-simple semigroups $I_{\alpha}$ indexed by the elements of $X$ and partial homomorphisms $f_{\alpha, \beta}$ between certain of the non zero parts of the semigroups $I_{\alpha}$. A semigroup so constructed is a tree of 0-simple semigroups. In [2] then it is shown that a semigroup with atomistic congruence lattice is an inflation of a tree of 0 -simple semigroups. In addition, for a weakly reductive tree of 0 -simple semigroups, necessary and sufficient conditions in order that its congruence lattice be atomistic are obtained. The purpose of this paper is to extend this characterization to the class of all semigroups.

For the remainder of this section we collect some definitions and results needed for our considerations. In Section 2 we obtain necessary and suffi-

(C) 1992 Australian Mathematical Society 0263-6115/92 \$A2.00+0.00 
cient conditions for a semigroup $S$ to have an atomistic congruence lattice. In section 3 we study a complete congruence $D$ on the congruence lattice of $S$. The greatest and least elements of the $D$-classes are determined. Furthermore, these classes coincide with the respective intervals in the lattice of all equivalence relations on $S$.

A semilattice $Y$ is a (locally finite) tree if each interval of the form $[x, y]=\{z \in Y \mid x \leq z \leq y\}$ is a (finite) chain. For a semigroup $S$, $S^{*}=S$ if $S$ has no zero and $S^{*}=S \backslash\{0\}$ if 0 is the zero of $S$. Let $S$ be a subsemigroup of a semigroup $T$. Then $T$ is an inflation of $S$ if there exists a function $f: T \rightarrow S$ such that $f \mid S=i d_{S}$ and $a b=(a f)(b f)$ for all $a, b \in T$. In this case $f$ is an inflation function. A semigroup $S$ is weakly reductive if for $a, b \in S, z a=z b$ and $a z=b z$ for all $z \in S$ imply $a=b$. A semigroup $S$ is globally idempotent if $S^{2}=S$. If $X$ is a partially ordered set then for $x, y \in X$ we say that $y$ covers $x$ or $x$ is covered by $y$, to be denoted by $y \succ x$ or $x \prec y$ if $x<y$ and $x<z \leq y$ imply $z=y$. The lattice of all congruences on a semigroup $S$ is denoted by $\mathscr{C}(S)$. The identical and the universal relations on $S$ are denoted by $\varepsilon=\varepsilon_{S}$ and $\omega=\omega_{S}$, respectively. A congruence $\rho$ on $S$ is an atom in $\mathscr{E}(S)$ if it covers $\varepsilon_{S}$. The set of all atoms of $\mathscr{C}(S)$ is denoted by $A t(S)$. For an arbitrary set $X$ let $\mathscr{E}(X)$ be the lattice of all equivalence relations on $X$ and $\mathscr{P}(X)$ the power set lattice of $X$. For an arbitrary binary relation $R$ on $S$, $R^{*}$ denotes the congruence generated by $R$, that is, the least congruence on $S$ which contains $R$.

For 0-simple semigroups we have the following

RESULT 1 ([3]). A 0-simple semigroup is congruence free if and only if for any two distinct elements $x, y \in S$ there exist $u, v \in S$ such that either $u x v=0$ and $u y v \neq 0$ or $u x v \neq 0$ and $u y v=0$.

The most important tool to describe the structure of semigroups with atomistic congruence lattice is the following.

Construction. Let $X$ be a locally finite tree. To each $\alpha \in X$ associate a 0 -simple semigroup $I_{\alpha} \neq\{0\}$ so that $I_{\alpha} \cap I_{\beta}=\varnothing$ if $\alpha \neq \beta$. For $\alpha \in X^{*}$ let $f_{\alpha}: I_{\alpha}^{*} \rightarrow I_{\alpha \prime}^{*}$ be a partial homomorphism where $\alpha^{\prime}$ denotes the unique element of $X$ which is covered by $\alpha$. Now let $f_{\alpha, \alpha}=i d_{I_{\alpha}^{*}}$ and $f_{\alpha, \beta}=f_{\alpha_{1}} f_{\alpha_{2}} \ldots f_{\alpha_{n}}$ where the elements $\alpha_{i}$ are defined by $\alpha=\alpha_{1} \succ \alpha_{2} \cdots \succ \alpha_{n} \succ \beta$. Suppose that for arbitrary $a \in I_{\alpha}^{*}$ and $b \in I_{\beta}^{*}$ the set

$$
\Delta(a, b)=\left\{\gamma \in X \mid\left(a f_{\alpha, \gamma}\right)\left(b f_{\beta, \gamma}\right) \text { is defined in } I_{\gamma}^{*}\right\}
$$


is not empty. Let $\delta(a, b)$ denote the greatest element of $\Delta(a, b)$. Then let $S=\bigcup\left\{I_{\alpha}^{*} \mid \alpha \in X\right\}$ and define a multiplication $*$ on $S$ by the rule

$$
a * b=\left(a f_{\alpha, \delta(a, b)}\right)\left(b f_{\beta, \delta(a, b)}\right) \quad\left(a \in I_{\alpha}^{*}, b \in I_{\beta}^{*}\right)
$$

where the right hand side product is computed in $I_{\delta(a, b)}^{*}$.

DEFINITION. The groupoid $S$ of the construction is a tree of 0 -simple semigroups to be denoted by $S=\left(X ; I_{\alpha}, f_{\alpha, \beta}\right)$. If each 0-simple semigroup $I_{\alpha}$, $\alpha \in X$, is congruence free then $S$ is a tree of congruence free semigroups.

If $X$ has a least element $\mu$ then by definition $I_{\mu}^{*}$ is closed under multiplication and thus is a simple semigroup. If, in addition, $S$ is a tree of congruence free semigroups then the congruence freeness of $I_{\mu}^{*} \cup\{0\}$ implies that $I_{\mu}^{*}$ consists of exactly one element. A straightforward verification shows that $S$ is a semigroup. This construction also appears in [1] and [2], a similar one in [4]. By the latter paper it follows that each ideal of such a semigroup is a retract.

The importance of this construction for the characterization of the semigroups under study is given by the following results.

Result 2 [2, Lemma 6, Theorem 1]. Let $S$ be a semigroup with atomistic congruence lattice. Then $S$ is an inflation of $S^{2}$ and $S^{2}$ is a tree of 0-simple semigroups.

Result 3 [2, Theorem 2]. Let $S$ be a weakly reductive semigroup. Then $\mathscr{C}(S)$ is atomistic if and only if $S$ is isomorphic to one of the following:

(1) a simple semigroup $I$ such that $\mathscr{C}(I)$ is atomistic,

(2) a tree of congruence free semigroups $\left(X ; I_{\alpha}, f_{\alpha, \beta}\right)$ such that for each $x \in I_{\alpha}^{*}, y \in I_{\beta}^{*}$ there exists $\gamma \leq \alpha, \beta$ satisfying $x f_{\alpha, \gamma}=y f_{\beta, \gamma}$,

(3) a tree of 0 -simple semigroups $\left(X ; I_{\alpha}, f_{\alpha, \beta}\right)$ where $X$ has a least element $\mu$ such that $I_{\mu}^{*}$ is a semigroup of type (1) and $S / I_{\mu}^{*}$ is a semigroup of type (2).

\section{Necessary and sufficient conditions for the not weakly reductive case}

We first consider the globally idempotent case. If $\mathscr{C}(S)$ is atomistic for the globally idempotent semigroup $S$ then by Result $2, S=\left(X ; I_{\alpha}, f_{\alpha, \beta}\right)$, a tree of 0 -simple semigroups $I_{\alpha}$. The goal is to derive necessary and sufficient 
conditions for the mappings $f_{\alpha}$ and $f_{\alpha, \beta}$, respectively, and the semigroups $I_{\alpha}$ in order that $\mathscr{C}(S)$ be atomistic. We first need the following

DEFINITION. On the partial semigroups $I_{\alpha}^{*}$ let $\tau_{\alpha}$ and $\delta_{\alpha}$, respectively, be the following equivalence relations:

$$
\begin{gathered}
x \tau_{\alpha} y \Longleftrightarrow\left(u x v \in I_{\alpha}^{*} \Leftrightarrow u y v \in I_{\alpha}^{*} \quad \forall u, v \in I_{\alpha}^{*}\right), \\
x \delta_{\alpha} y \Longleftrightarrow z x=z y \text { and } x z=y z \quad \forall z \in I_{\alpha}^{*} .
\end{gathered}
$$

In the definition of $\delta_{\alpha}$ equality means that if one side is defined in $I_{\alpha}^{*}$ then the other is also and both sides are equal. Obviously we have $\delta_{\alpha} \subseteq \tau_{\alpha}$. Furthermore, $\tau_{\alpha} \cup\left\{\left(0_{\alpha}, 0_{\alpha}\right)\right\}$ is the greatest non universal congruence on $I_{\alpha}$. If $\rho_{\alpha}$ denotes the greatest congruence on $S$ which saturates $I_{\alpha}^{*}$, that is, the greatest congruence $\rho$ on $S$ such that $I_{\alpha}^{*}$ is a union of $\rho$-classes, then $\rho_{\alpha} \mid I_{\alpha}^{*}=\tau_{\alpha}($ see $[2$, Lemma 8]).

LemMA 1 [1, Lemma 9]. Let $S=\left(X ; I_{\alpha}, f_{\alpha, \beta}\right)$ be a tree of 0-simple semigroups, $\alpha \geq \beta \geq \gamma \geq \delta \in X, \rho \in \mathscr{C}(S)$ and $x \rho y$ for some $x \in I_{\alpha}^{*}$ and $y \in I_{\delta}^{*}$. Then $z \rho z f_{\beta, \gamma}$ for all $z \in I_{\beta}^{*}$.

In the following statements let the congruence lattice of $S=\left(X ; I_{\alpha}, f_{\alpha, \beta}\right)$ be atomistic.

LemMa 2 [2, Lemma 8]. Let $\alpha \in X^{*}$. If $x \tau_{\alpha} y$ and $x f_{\alpha}=y f_{\alpha}$ then $x=y$. In particular, the restriction of $f_{\alpha}$ to an arbitrary $\tau_{\alpha}$-class is injective.

LemMA 3. Let $\alpha \in X^{*}$ and $x, y \in I_{\alpha}^{*}$. If $x \tau_{\alpha} y$ then $x f_{\alpha, \beta} \delta_{\beta} y f_{\alpha, \beta}$ for all $\beta<\alpha$. In particular,

$$
\left(x \tau_{\alpha}\right) f_{\alpha, \beta} \subseteq\left(x f_{\alpha, \beta}\right) \delta_{\beta} \subseteq\left(x f_{\alpha, \beta}\right) \tau_{\beta} \quad \text { for all } \beta<\alpha .
$$

Proof. Let $x, y \in I_{\alpha}^{*}$ be such that $x \tau_{\alpha} y$. Then by [2, Lemma 8] $x \rho_{\alpha} y$ where $\rho_{\alpha}$ is the greatest congruence which saturates $I_{\alpha}^{*}$. Now there exist $\rho_{1}, \rho_{2} \ldots \rho_{n} \in A t(S)$ and $z_{0}, z_{1} \ldots z_{n} \in S$ such that $x=z_{0} \rho_{1} z_{1} \rho_{2}$ $z_{2} \ldots \rho_{n} z_{n}=y$ and $\rho_{i} \subseteq \rho_{\alpha}$ for all $i$. The congruences $\rho_{i}$ saturate $I_{\alpha}^{*}$. Thus we may assume that $z_{i} \in I_{\alpha}^{*}$ and $z_{i} \rho_{i} \neq\left\{z_{i}\right\}$ for all $i$. Since each $\rho_{i}$ is an atom we conclude that $\rho_{i} \mid I_{\beta}^{*}=\varepsilon_{I_{\beta}^{*}}$ for all $\beta<\alpha$. Now let $z \in I_{\beta}^{*}$ for some $\beta<\alpha$. Multiplying the above sequence by $z$ from the right and the left, respectively, yields

$$
\left(x f_{\alpha, \beta}\right) z=x z=\cdots=y z=\left(y f_{\alpha, \beta}\right) z
$$


and

Hence $x f_{\alpha, \beta} \delta_{\beta} y f_{\alpha, \beta}$.

$$
z\left(x f_{\alpha, \beta}\right)=z x=\cdots=z y=z\left(y f_{\alpha, \beta}\right) .
$$

COROllary 1. If $\alpha$ is not minimal in $X$ then $\tau_{\alpha}=\delta_{\alpha}$.

Proof. Let $x, y, z \in I_{\alpha}^{*}$ be such that $x \tau_{\alpha} y$ and $x z, z x \in I_{\alpha}^{*}$. Then also $y z, z y \in I_{\alpha}^{*}$. Let $\beta<\alpha$. By Lemma 3,

$$
(x z) f_{\alpha, \beta}=x f_{\alpha, \beta} z f_{\alpha, \beta}=y f_{\alpha, \beta} z f_{\alpha, \beta}=(y z) f_{\alpha, \beta}
$$

and

$$
(z x) f_{\alpha, \beta}=z f_{\alpha, \beta} x f_{\alpha, \beta}=z f_{\alpha, \beta} y f_{\alpha, \beta}=(z y) f_{\alpha, \beta} .
$$

Also, $x \tau_{\alpha} y$ implies $x z \tau_{\alpha} y z$ and $z x \tau_{\alpha} z y$. By Lemmas 2 and 3 we obtain that $x z=y z$ and $z x=z y$.

LemMa 4. Let $\rho \in \mathscr{C}(S)$. Then $\rho$ is an atom in $\mathscr{C}(S)$ if and only if either $\rho$ is an atom that saturates $I_{\alpha}^{*}$ for all $\alpha \in X$ or there exists an $\alpha \in X^{*}$ such that $\rho=\left(\varepsilon \cup f_{\alpha}\right)\left(\varepsilon \cup f_{\alpha}^{-1}\right)$.

Proof. Let $\rho$ be an atom of $\mathscr{C}(S)$ which does not saturate each $I_{\alpha}^{*}$. There exist $x \in I_{\alpha}^{*}, y \in I_{\beta}^{*}$ for some $\alpha \neq \beta$ such that $x \rho y$. We may assume that $\alpha \not \leq \beta$. Let $z \in I_{\alpha}^{*}$ be such that $z x \in I_{\alpha}^{*}$. Then $z x \rho z y$ and $z y \in I_{\gamma}^{*}$ for some $\gamma<\alpha$. By Lemma 1 we obtain that $u \rho u f_{\alpha}$ for all $u \in I_{\alpha}^{*}$. Now let $\xi=\left(\varepsilon \cup f_{\alpha}\right)\left(\varepsilon \cup f_{\alpha}^{-1}\right)$. It can be seen easily that $\xi$ is a congruence on $S$. Since $\rho$ is an atom, $\xi \subseteq \rho$ implies $\rho=\xi$.

Lemma 5. Let $\alpha \in X$. For $x, y \in I_{\alpha}^{*}$ there exists $\gamma \leq \alpha$ such that $x f_{\alpha, \gamma} \tau_{\gamma} y f_{\alpha, \gamma}$.

Proof. Let $x, y \in I_{\alpha}^{*}$. We consider the congruence generated by the elements $x$ and $y$, to be denoted by $(x, y)^{*}$. There exist atoms $\rho_{i} \subseteq$ $(x, y)^{*}, 1 \leq i \leq n$, and $z_{0}, z_{1}, \ldots z_{n}$ such that $x=z_{0} \rho_{1} z_{1} \rho_{2} z_{2} \ldots \rho_{n}$ $z_{n}=y$ and we may assume that $n=n(x, y)$ is the shortest possible length of such a sequence. If $n=1$ then by Lemma 4 either $x \tau_{\alpha} y$ or $x f_{\alpha}=y f_{\alpha}$. Let $n>1$ and suppose that the assertion is proved for arbitrary elements $u, v \in I_{\beta}^{*}$ and arbitrary $\beta \in X$ for which $n(u, v)<n$. If $x \tau_{\alpha} y$ then nothing has to be proved. Let $z_{i} \in I_{\alpha_{i}}^{*}$. Then $\rho_{i} \subseteq(x, y)^{*}$ implies $\alpha_{i} \leq \alpha$ for all $i$. If $\alpha_{i}=\alpha$ for all $i$ then $x \tau_{\alpha} y$. If not then let $i$ and $j$ be the least and the greatest indices, respectively, such that $\alpha_{i} \neq \alpha \neq \alpha_{j}$. Then by Lemma $4 \alpha_{i}=\alpha_{j}=\alpha^{\prime}$, the unique element of $X$ which is covered by 
$\alpha$. By hypothesis there exists $\gamma \leq \alpha^{\prime}$ such that $z_{i} f_{\alpha \prime, \gamma} \tau_{\gamma} z_{j} f_{\alpha \prime, \gamma}$. Then $z_{i}=z_{i-1} f_{\alpha}$ and $z_{j}=z_{j+1} f_{\alpha}$ and so $z_{i-1} f_{\alpha, \gamma}=z_{i-1} f_{\alpha} f_{\alpha \prime, \gamma}=z_{i} f_{\alpha \prime, \gamma}$ and $z_{j} f_{\alpha \prime, \gamma}=z_{j+1} f_{\alpha} f_{\alpha \prime, \gamma}=z_{j+1} f_{\alpha, \gamma}$. Furthermore, $x \tau_{\alpha} z_{i-1}$ and $z_{j+1} \tau_{\alpha} y$. By Lemma 3 we have $x f_{\alpha, \gamma} \tau_{\gamma} z_{i-1} f_{\alpha, \gamma}$ and $z_{j+1} f_{\alpha, \gamma} \tau_{\gamma} y f_{\alpha, \gamma}$. Hence $x f_{\alpha, \gamma} \tau_{\gamma} y f_{\alpha, \gamma}$.

From these results we obtain the following consequence for semigroups without kernel.

Proposition 1. If $X$ has no least element then $\tau_{\alpha}=\varepsilon_{I_{\alpha}^{*}}$ for all $\alpha \in X$. In particular, all semigroups $I_{\alpha}$ are congruence free.

Proof. First, by Corollary 1, $\tau_{\alpha}=\delta_{\alpha}$ for all $\alpha \in X$. Let $\sigma$ be the least simple congruence on $S$. Then $x \sigma y$ if and only if $x f_{\alpha, \gamma}=y f_{\beta, \gamma}$ for some $\gamma \leq \alpha, \beta\left(x \in I_{\alpha}^{*}, y \in I_{\beta}^{*}\right)$. Let $x \in I_{\alpha}^{*}, y \in I_{\beta}^{*}, z \in I_{\gamma}^{*}$. Then there exists $\nu \leq \alpha, \beta$ such that $x f_{\alpha, \nu} \delta_{\nu} y f_{\beta, \nu}$. Let $\lambda<\alpha, \beta, \gamma, \nu$. Then

$$
\left(x f_{\alpha, \lambda}\right)\left(z f_{\gamma, \lambda}\right)=\left(y f_{\beta, \lambda}\right)\left(z f_{\gamma, \lambda}\right)
$$

and

$$
\left(z f_{\gamma, \lambda}\right)\left(x f_{\alpha, \lambda}\right)=\left(z f_{\gamma, \lambda}\right)\left(y f_{\beta, \lambda}\right) .
$$

In $S / \sigma$ therefore $x \sigma y \sigma=u \sigma v \sigma$ holds for arbitrary $x \sigma, y \sigma, u \sigma, v \sigma \in S / \sigma$. Since $S / \sigma$ is globally idempotent this implies that $|S / \sigma|=1$ and so $\sigma=\omega$. But if $x \delta_{\alpha} y$ for some distinct elements $x, y \in I_{\alpha}^{*}$ then $x f_{\alpha, \beta} \neq y f_{\alpha, \beta}$ for all $\beta<\alpha$. Hence $\delta_{\alpha}=\tau_{\alpha}=\varepsilon_{I_{\alpha}^{*}}$. Now by Result 1 each $I_{\alpha}$ is congruence free.

Proposition 2. If $S$ has a weakly reductive kernel, that is, $X$ has a least element $\mu$ and $I_{\mu}^{*}$ is a weakly reductive semigroup then $\tau_{\alpha}=\varepsilon_{I_{\alpha}^{*}}$ for all $\alpha \in X^{*}$.

Proof. Let $\alpha \in X^{*}$ and $x \tau_{\alpha} y$. Then by Lemma 3, $x f_{\alpha, \mu} \delta_{\mu} y f_{\alpha, \mu}$. Since $I_{\mu}^{*}$ is weakly reductive $x f_{\alpha, \mu}=y f_{\alpha, \mu}$. By Lemmas 2 and 3 then $x=y$.

In other words, if $S=\left(X ; I_{\alpha}, f_{\alpha, \beta}\right)$ either has a weakly reductive kernel or no kernel at all then $S$ is weakly reductive and we may apply the results of [2]. We still have to identify those inflations of $S$ which have atomistic congruence lattices. 
DEFINITION. Let $T$ be an inflation of a semigroup $S$ with inflation function $f$. Then $f$ is trivial if $|(T \backslash S) f|=1$.

Roughly speaking, $f$ is trivial if only one element of $S$ is inflated by $f$.

LeMMA 6. Let $S$ be weakly reductive and $T$ be an inflation of $S$. If $\mathscr{C}(T)$ is atomistic then the inflation function $f$ is trivial.

Proof. We consider the congruence $\xi$, defined by

$$
x \xi y \Leftrightarrow\{x, y\} \subseteq S \text { or }\{x, y\} \subseteq T \backslash S .
$$

Then $\xi$ is the supremum of atoms which saturate the sets $S$ and $T \backslash S$. Let $x, y \in T \backslash S$. Then $x=z_{0} \rho_{1} z_{1} \ldots \rho_{n} z_{n}=y$ for certain $z_{i} \in T$ and $\rho_{i} \in A t(S), \rho_{i} \subseteq \xi$. Since each $\rho_{i}$ saturates $T \backslash S$ it follows that $z_{i} \in T \backslash S$ for all $i$. Since $\rho_{i}$ is an atom we have $\rho_{i} \mid S=\varepsilon_{S}$. For arbitrary $z \in S$ we obtain that $z z_{i}=z z_{i+1}$ and $z_{i} z=z_{i+1} z$ for all $i$ and thus $z x=z(x f)=z(y f)=z y$ and $x z=(x f) z=(y f) z=y z$. By weak reductivity of $S$ then $x f=y f$.

LEMMA 7. If $S$ is weakly reductive and $\mathscr{C}(S)$ atomistic then $S / \rho$ is weakly reductive for each $\rho \in \mathscr{C}(S)$

Proof. Let $\rho \in \mathscr{C}(S), x, y \in S$ and suppose that $z x \rho z y$ and $x z \rho y z$ for all $z \in S$. Let $\xi=\rho \cap(x, y)^{*}$. If $\xi \subsetneq(x, y)^{*}$ then there exists an atom $\eta \in \operatorname{At}(S)$ such that $\eta \subseteq(x, y)^{*}$ but $\eta \nsubseteq \xi$. Let $u \eta v$ and $u \neq v$. Then $u(x, y)^{*} v$ and hence $z u \xi z v$ and $u z \xi v z$ for all $z \in S$. Since $\xi \cap \eta=\varepsilon$ we have $z u=z v$ and $u z=v z$ for all $z \in S$ which is a contradiction to weak reductivity.

We therefore may apply

Lemma 8 [2, Proposition 4]. Let $T$ be an inflation of a semigroup $S$ such that each homomorphic image of $S$ is weakly reductive. Then $\mathscr{C}(T)$ is atomistic if and only if $\mathscr{C}(S)$ is atomistic and the inflation function $f$ is trivial.

We thus obtain using Result 3

THEOREM 1. Let $S$ be a semigroup which has either a weakly reductive kernel or no kernel at all. Then $\mathscr{C}(S)$ is atomistic if and only if $S$ is one of the following:

(1) a simple semigroup $I$ with atomistic congruence lattice $\mathscr{C}(I)$, 
(2) a tree of congruence free semigroups $\left(X ; I_{\alpha}, f_{\alpha, \beta}\right)$ such that for $x \in$ $I_{\alpha}^{*}, y \in I_{\beta}^{*}$ there exists $\gamma \leq \alpha, \beta$ so that $x f_{\alpha, \gamma}=y f_{\beta, \gamma}$,

(3) a tree of 0 -simple semigroups $\left(X ; I_{\alpha}, f_{\alpha, \beta}\right)$ such that $X$ has a least element $\mu, I_{\mu}^{*}$ is of type (1) and $S / I_{\mu}^{*}$ is of type (2),

(4) an inflation of a semigroup of types (1),(2) or (3) with a trivial inflation function $f$.

DEFINITION. A congruence $\rho$ on a semigroup $S$ is weakly reductive if $S / \rho$ is a weakly reductive semigroup.

LEMMA 9. Let $S$ be an inflation of a globally idempotent semigroup. Then

$$
\delta=\delta_{S}=\{(x, y) \mid x z=y z, z x=z y \quad \forall z \in S\}
$$

is the least weakly reductive congruence on $S$.

PROOF. The proof is straightforward.

Semigroups $S$ with the property that each homomorphic image of $S$ has an atomistic congruence lattice now can be described easily.

COROLlary 2. Let $S$ be a semigroup. Then each homomorphic image of $S$ has an atomistic congruence lattice if and only if $S$ is one of the following:

(1) a simple semigroup I such that each homomorphic image of I has an atomistic congruence lattice,

(2) a tree of congruence free semigroups $\left(X ; I_{\alpha}, f_{\alpha, \beta}\right)$ such that for $x \in$ $I_{\alpha}^{*}, y \in I_{\beta}^{*}$ there exists $\gamma \leq \alpha, \beta$ so that $x f_{\alpha, \gamma}=y f_{\beta, \gamma}$,

(3) a tree of 0 -simple semigroups $\left(X ; I_{\alpha}, f_{\alpha, \beta}\right)$ such that $X$ has a least element $\mu, I_{\mu}^{*}$ is of type (1) and $S / I_{\mu}^{*}$ is of type (2),

(4) an inflation of a semigroup of types (1),(2) or (3) with a trivial inflation function $f$.

Proof. Necessity. Suppose each homomorphic image of the semigroup $S$ has an atomistic congruence lattice. If $S$ has no kernel then $S$ is of type (2) or an inflation thereof with trivial inflation function. Suppose that $S$ has a kernel $I$. Since $I$ is a retract ideal each homomorphic image of $I$ has an atomistic congruence lattice. We have to show that $I$ is weakly reductive. Let $K$ be a subdirectly irreducible homomorphic image of $I$. Then $\mathscr{C}(K)$ is atomistic. On the other hand, $\mathscr{C}(K)$ has exactly one atom since $K$ is subdirectly irreducible. Hence $K$ is congruence free. If $\delta_{K}=\omega$ then $K$ is a null semigroup which is impossible since $K$ is simple. Hence $K$ is 
weakly reductive. Therefore, $I$ is a subdirect product of weakly reductive semigroups and hence itself is weakly reductive.

Sufficiency. It is clear that a semigroup of type (1) has the desired property. For semigroups $S$ of types (2)-(4) it can be seen easily that each homomorphic image of $S$ is again of the respective type or a less complicated one. Then by Theorem 1 each homomorphic image of $S$ has an atomistic congruence lattice. (See also Theorem 3 of the next section where an alternative proof for this part is given).

It remains to treat the problem for semigroups $S$ which do have a non weakly reductive kernel.

LEMMA 10. Let $T$ be an inflation of a semigroup $S$ with inflation function f. If $\mathscr{C}(T)$ is atomistic then $(a f) \delta_{S}(b f)$ for all $a, b \in T \backslash S$.

Proof. This is proved in the same way as Lemma 6.

LEMMA 11. Let $S=\left(X ; I_{\alpha}, f_{\alpha, \beta}\right)$ be such that $\left(x \delta_{\alpha}\right) f_{\alpha, \beta} \subseteq\left(x f_{\alpha, \beta}\right) \delta_{\beta}$ for all $\alpha \geq \beta \in X, x \in I_{\alpha}^{*}$. Then $\delta_{\alpha} \subseteq \delta_{S}$ for each $\alpha \in X$.

Proof. Let $x \delta_{\alpha} y, z \in I_{\beta}^{*}, x z \in I_{\gamma}^{*}$ and $y z \in I_{\nu}^{*}$. Then $\gamma$ and $\nu$ are comparable. We assume $\gamma \geq \nu$. By hypothesis, $\left(x f_{\alpha, \gamma}\right) \delta_{\gamma}\left(y f_{\alpha, \gamma}\right)$ and hence

$$
x z=\left(x f_{\alpha, \gamma}\right)\left(z f_{\beta, \gamma}\right)=\left(y f_{\alpha, \gamma}\right)\left(z f_{\beta, \gamma}\right) \in I_{\gamma}^{*} \text {. }
$$

This implies that $\gamma=\nu$ and thus $x z=y z$. Dually also $z x=z y$.

Recall the definitions of the relations $\delta_{\alpha}$ and $\tau_{\alpha}$ given at the beginning of this section, and of the mapping $f_{\alpha}$ on a tree of 0 -simple semigroups $\left(X ; I_{\alpha}, f_{\alpha, \beta}\right)$. The remaining case then is answered by

THEOREM 2. Let $S$ be a semigroup subject to the following conditions:

(1) $S$ is an inflation of $S^{2}$ with inflation function $f$,

(2) $S^{2}=\left(X ; I_{\alpha}, f_{\alpha, \beta}\right)$, a tree of 0-simple semigroups,

(3) $X$ has a least element $\mu$,

(4) $\mathscr{C}\left(I_{\mu}^{*}\right)$ is atomistic,

(5) $\delta_{\alpha}=\tau_{\alpha}$ for $\alpha \in X^{*}$

(6) $\left(x \delta_{\alpha}\right) f_{\alpha} \subseteq\left(x f_{\alpha}\right) \delta_{\alpha \prime}$ for $x \in I_{\alpha}^{*}, \alpha \in X^{*}$,

(7) if $x \delta_{\alpha} y$ and $x f_{\alpha}=y f_{\alpha}$ then $x=y$ for $x, y \in I_{\alpha}^{*}, \alpha \in X^{*}$,

(8) (af) $\delta_{S^{2}}(b f)$ for all $a, b \in S \backslash S^{2}$,

(9) if $x \in S \backslash S^{2}, y \in S^{2}, y \notin(x f) \delta_{S^{2}}$ then there exists $z \in(x f) \delta_{S^{2}}$ such that $(z, y) \in\left\{(u z, u y),(z u, y u) \mid u \in S^{2}\right\}^{*}$. 
Then $\mathscr{C}(S)$ is atomistic. Conversely, each semigroup $S$ with kernel $I_{\mu}^{*}$ and atomistic congruence lattice $\mathscr{C}(S)$ can be so constructed.

Proof. Suppose $S$ has an atomistic congruence lattice. If $S$ has a kernel $I$ then by Result $2 I$ is a retract ideal. Thus

$$
\mathscr{C}(I) \cong\left[\varepsilon, \rho_{I}\right] \subseteq \mathscr{C}(S)
$$

where $\rho_{I}$ denotes the Rees congruence with respect to $I$. Therefore $\mathscr{C}(I)$ is atomistic. Hence the necessity of the conditions (1)-(8) is obtained by Results 2,3, Lemmas 2,3,5,10 and Corollary 1. Now let $x \in S \backslash S^{2}, y \in S^{2}$ and $(y, x f) \notin \delta_{S^{2}}$. The congruence $(x, y)^{*}$ is a supremum of atoms. For $1 \leq i \leq n$ let $\rho_{i} \in \operatorname{At}(S), \rho_{i} \subseteq(x, y)^{*}, x_{i} \in S$ be such that

$$
x \rho_{1} x_{1} \rho_{2} \cdots \rho_{n} x_{n}=y .
$$

If $k$ is the least index such that $x_{k} \in S^{2}$ then

$$
(x f) \delta_{S^{2}} \ldots\left(x_{k-1} f\right) \delta_{S^{2}} x_{k} \text {. }
$$

Let $z=x_{k}$. Then we have $(z, y) \in(x, y)^{*}$. Since $x$ cannot be written as a product we obtain that $(z, y) \in\{(x u, y u),(u x, u y) \mid u \in S\}^{*}$ and hence $(z, y) \in\left\{(z u, y u),(u z, u y) \mid u \in S^{2}\right\}^{*}$ since $u x=u(x f)=u z$ and $x u=(x f) u=z u$ for all $u \in S^{2}$ which proves the necessity of condition (9).

In order to prove sufficiency we show that each congruence of the form $(x, y)^{*}$ is a supremum of atoms. Let $x, y \in S$. If $x, y \in S \backslash S^{2}$ then by condition $(8)(x, y)^{*}=\{(x, y),(y, x)\} \cup \varepsilon_{S}$ which trivially itself is an atom. If $x \in S \backslash S^{2}$ and $y \in S^{2}$ then by condition (9) there exists $z \in(x f) \delta_{S^{2}}$ such that $(z, y) \in(x, y)^{*}$. Then $\{(z, x),(x, z)\} \cup \varepsilon_{S}$ is an atom contained in $(x, y)^{*}$. Hence it remains to show that $(z, y)^{*}$ is a supremum of atoms for arbitrary $z, y \in S^{2}$. Let $z \in I_{\alpha}^{*}, y \in I_{\beta}^{*}$ and $\rho=(z, y)^{*}$. We have, using Lemma 1, that $a \rho a f_{\alpha, \alpha \beta}$ and $b \rho b f_{\beta, \alpha \beta}$ for all $a \in I_{\alpha}^{*}, b \in I_{\beta}^{*}$. Let $u=z f_{\alpha, \alpha \beta}$ and $v=y f_{\beta, \alpha \beta}$. Suppose that $(u, v) \notin \tau_{\alpha \beta}$. Then by definition there exist $r, s \in I_{\alpha \beta}^{*}$ such that rus $\in I_{\alpha \beta}^{*}$ and $r v s \notin I_{\alpha \beta}^{*}$, or conversely. In any case, by Lemma 1 we obtain that $t \rho t f_{\alpha \beta}$ for all $t \in I_{\alpha \beta}^{*}$ and $u f_{\alpha \beta} \rho v f_{\alpha \beta}$. Now either $u f_{\alpha \beta} \tau_{(\alpha \beta)} v f_{\alpha \beta}$ or we may apply the same procedure to the pair $\left(u f_{\alpha \beta}, v f_{\alpha \beta}\right)$. Iterating this process we obtain that $u f_{\alpha \beta, \gamma} \rho u \rho v \rho v f_{\alpha \beta, \gamma}$ where $\gamma$ denotes the greatest element of $X$ such that $u f_{\alpha \beta, \gamma} \tau_{\gamma} v f_{\alpha \beta, \gamma}$. Since $\tau_{\mu}=\omega_{I_{\mu}^{*}}$ the element $\gamma$ surely exists (and is well defined). For $\lambda \in X^{*}$ denote by $\phi_{\lambda}$ the atom $\phi_{\lambda}=\left(\varepsilon_{S} \cup\right.$ $\left.f_{\lambda}\right)\left(\varepsilon_{S} \cup f_{\lambda}^{-1}\right)$. Then $\left(z, z f_{\alpha, \gamma}\right) \in \bigvee_{\alpha \geq \lambda>\gamma} \varphi_{\lambda}$ and analogously, $\left(y, y f_{\beta, \gamma}\right) \in$ $\bigvee_{\beta \geq \lambda>\gamma} \varphi_{\lambda}$. Also, the atoms $\phi_{\lambda}$ are contained in $(z, y)^{*}$ if $\alpha \geq \lambda>\gamma$ and 
$\beta \geq \lambda>\gamma$. If $\gamma>\mu$ then by condition (5) and Lemma 11 we recognize that $\left\{\left(z f_{\alpha, \gamma}, y f_{\beta, \gamma}\right),\left(y f_{\beta, \gamma}, z f_{\alpha, \gamma}\right)\right\} \cup \varepsilon_{S}$ is an atom in $\mathscr{E}(S)$ which is contained in $(z, y)^{*}$. If $\gamma=\mu$ then there exist atoms $\rho_{1}, \rho_{2}, \ldots \rho_{n} \in \mathscr{C}\left(I_{\mu}^{*}\right)$ such that $\rho_{i} \subseteq\left(z f_{\alpha, \gamma}, y f_{\beta, \gamma}\right)^{*}$ and $\left(z f_{\alpha, \gamma}, y f_{\beta, \gamma}\right) \in \bigvee_{i=1}^{n} \rho_{i}$. The congruences $\rho_{i} \cup \varepsilon_{S}$ then are atoms in $\mathscr{C}(S)$ and contained in $(z, y)^{*}$. In any case, we have found (a finite number of) atoms $\psi_{i} \subseteq(z, y)^{*}$ such that $z \bigvee \psi_{i} y$.

Problems. (1) Are the semigroups with kernel characterized by Theorem 1 a proper subclass of those given by Theorem 2? In order to answer this question it is sufficient to prove (the impossibility of) the existence of a non weakly reductive, simple semigroup whose congruence lattice is atomistic.

(2) Can the conditions (5)-(7) in Theorem 2 be replaced by " $I_{\alpha}$ is congruence free for all $\alpha \in X^{*}$ "? A negative answer of (1) implies a positive answer of (2).

\section{Decompositions of the congruence lattice}

In this section we study a complete congruence on an atomistic congruence lattice which decomposes it into a disjoint union of intervals. These are intervals in the lattice of all equivalence relations of $S$. The situation for semigroups with weakly reductive kernel or without kernel is described in

Theorem 3. Let $S$ be a semigroup characterized by Theorem 1. Then

$$
\mathscr{C}(S) \cong \mathscr{E}\left(S / S^{2}\right) \times \mathscr{P}\left(X^{*}\right) \times \mathscr{C}(I)
$$

for the locally finite tree $X=S^{2} / \mathscr{J}$ and the simple semigroup $I=\operatorname{kernel}(S)$ or $I=\{0\}$ if $S$ has no kernel.

Proof. Let $x \in S \backslash S^{2}, y \in S^{2}$ and $\rho \in \mathscr{C}(S)$ be such that $x \rho y$. Then $(x f) z \rho y z$ and $z(x f) \rho z y$ for all $z \in S^{2}$ and hence $x f \rho y$ since by Lemma $7 S^{2} / \rho$ is weakly reductive. This ensures that the mapping $\rho \mapsto\left(\rho\left|S^{2}, \rho\right|\left[S \backslash S^{2} \cup\{x f\}\right]\right)$ is injective and thus is an isomorphism between $\mathscr{C}(S)$ and $\mathscr{C}\left(S^{2}\right) \times \mathscr{E}\left(S \backslash S^{2} \cup\{x f\}\right)$ where $x f$ denotes the unique element of $S^{2}$ which is inflated by $f$. Moreover, by [2, Lemmas 13-15] it follows that $\mathscr{C}\left(S^{2}\right) \cong \mathscr{P}\left(X^{*}\right)$ if $S^{2}$ has no kernel and $\mathscr{C}\left(S^{2}\right) \cong \mathscr{P}\left(X^{*}\right) \times \mathscr{C}(I)$ where $I$ is the kernel of $S^{2}$ if it exists.

This factorization immediately implies that for this kind of semigroup $\mathscr{C}(S / \rho)$ is atomistic for each $\rho \in \mathscr{C}(S)$ if and only if $\mathscr{C}(I / \xi)$ is atomistic for each $\xi \in \mathscr{C}(I)$. 
Now to the general case characterized by Theorem 2 . Let $S$ be a semigroup with atomistic congruence lattice $\mathscr{E}(S)$ and $\delta$ denote the congruence on $S$ given by

$$
x \delta x \Longleftrightarrow z x=z y \quad \text { and } \quad x z=y z \quad \forall z \in S .
$$

Definition. Let $\rho \in \mathscr{C}(S)$. Then $\operatorname{At}(\rho)=\{\eta \in \operatorname{At}(S) \mid \eta \subseteq \rho\}$ and $\mathscr{B}=\operatorname{At}(S) \backslash \operatorname{At}(\delta)=\{\rho \in \operatorname{At}(S) \mid \rho \nsubseteq \delta\}$.

Let $\rho \in \mathscr{C}(S)$. Then $\rho \in \operatorname{At}(\delta)$ if and only if $\rho=\{(x, y),(y, x)\} \cup$ $\varepsilon_{S}$ for $x \neq y$ and $x \delta y$. If $\mathscr{B}=\varnothing$ then $\delta=\omega$ so that $S$ is a null semigroup and hence an inflation of the trivial semigroup. Then $S$ satisfies the conditions of Theorems 1 and 3, respectively. Hence we may assume that $\mathscr{B} \neq \varnothing$. Let $\mathscr{C}$ denote the set of all congruences which are joins of arbitrary subsets of $\mathscr{B}$. In the next statements we prove that $\mathscr{B}$ is a lattice isomorphic to $\mathscr{C}(S / \delta)$.

Lemma 12. Let $G, H \subseteq \mathscr{B}$. Then

$$
\bigvee G \subseteq \bigvee H \Longleftrightarrow \delta \vee \bigvee G \subseteq \delta \vee \bigvee H
$$

Proof. The direct implication is trivial. For the converse, let $\rho \in G$. Then $\rho \subseteq \delta \vee \vee H$. Let $x \neq y$ be such that $x \rho y$. Then $x=z_{0} \xi_{1}$ $z_{1} \cdots \xi_{n} z_{n}=y$ for certain $z_{i} \in S$ and $\xi_{i}=\delta$ or $\xi_{i} \in H$. We obtain that $x z \vee H y z$ and $z x \vee H z y$ for all $z \in S$. Since $\rho \in \mathscr{B}$ there exists $z$ such that $x z \neq y z$ or $z x \neq z y$. Assume $x z \neq y z$. Now $\rho$ is an atom so $\rho=(x z, y z)^{*}$. Therefore $\rho \subseteq \bigvee H$ which implies that $\bigvee G \subseteq \bigvee H$.

We therefore obtain

Proposition 3. The mapping $d: \mathscr{C} \rightarrow \mathscr{C}(S / \delta)$ defined by $\rho \mapsto(\rho \vee$ $\delta) / \delta$ is an isomorphism between $\mathscr{C}$ and $\mathscr{C}(S / \delta)$. In particular, $\mathscr{C}(S / \delta)$ is atomistic. The atoms in $\mathscr{C}(S / \delta)$ are precisely the elements $(\rho \vee \delta) / \delta$ for $\rho \in \mathscr{B}$.

PROof. Since each congruence $\rho \in[\delta, \omega]$ is the supremum of the atoms contained in $\rho, d$ is surjective. By Lemma 12, $d$ is injective. Also by Lemma $12, d$ and its inverse $d^{-1}$ are monotone and so $d$ is a lattice isomorphism.

Notation. For arbitrary sets $A, B$ let $A \Delta B=A \cup B \backslash A \cap B=A \backslash B \cup B \backslash A$. 
Definition. For $\rho, \xi \in \mathscr{C}(S)$ let

$$
\rho D \xi \Longleftrightarrow A t(\rho) \Delta A t(\xi) \subseteq A t(\delta) .
$$

Roughly speaking, $\rho D \xi$ if the atoms in which these congruences are different are contained in $\delta$.

LEMMA 13. The relation $D$ is a complete $\bigcap$-congruence on $\mathscr{C}(S)$.

Proof. The relation $D$ clearly is reflexive and symmetric. Let $\rho D \xi$ and $\xi D \eta$. Then

$$
A t(\rho) \Delta A t(\eta) \subseteq A t(\rho) \Delta A t(\xi) \cup A t(\xi) \Delta A t(\eta) \subseteq A t(\delta)
$$

implies that $D$ is transitive. Now let $\left\{\rho_{i} \mid i \in I\right\}$ and $\left\{\xi_{i} \mid i \in I\right\}$ be subsets of $\mathscr{C}(S)$ such that $\rho_{i} D \xi_{i}$ for all $i \in I$. Let $\rho=\bigcap \rho_{i}$ and $\xi=\bigcap \xi_{i}$. If $\chi \in A t(\rho) \backslash A t(\xi)$ then $\chi \in A t\left(\rho_{i}\right)$ for all $i$ and $\chi \notin A t\left(\xi_{j}\right)$ for some $j \in I$. Then $\chi \in A t\left(\rho_{j}\right) \backslash A t\left(\xi_{j}\right)$ and hence $\chi \in A t(\delta)$. Dually also $\operatorname{At}(\xi) \backslash A t(\rho) \subseteq$ $A t(\delta)$.

The following lemma gives an alternative characterization of $D$.

LeMma 14. Let $\rho, \xi \in \mathscr{C}(S)$. Then $\rho D \xi$ if and only if $\rho \vee \delta=\xi \vee \delta$.

Proof. Let $\rho \vee \delta=\xi \vee \delta$ and suppose $(\rho, \xi) \notin D$. Then there exists $\eta \in \mathscr{B}$ such that $\eta \subseteq \rho$ and $\eta \nsubseteq \xi$ (or conversely). By $\rho \vee \delta=\xi \vee \delta$ we obtain $\eta \subseteq \xi \vee \delta$. Since $\eta \in \mathscr{B}$ by the same argument as in the proof of Lemma 12 we obtain $\eta \subseteq \xi$, a contradiction.

Conversely, let $\rho D \xi$. We shall prove that $\operatorname{At}(\rho \vee \delta)=A t(\xi \vee \delta)$. Let $\eta \in A t(\rho \vee \delta)$. If $\eta \in A t(\delta)$ then clearly $\eta \in A t(\xi \vee \delta)$. If $\eta \notin \operatorname{At}(\delta)$ then $\eta \in \mathscr{B}$ and by the same argument as in the proof of Lemma 12 we recognize that $\eta \subseteq \rho$ and hence $\eta \in A t(\rho)$. Then $\rho D \xi$ implies that $\eta \in A t(\xi)$ and therefore, $\eta \in A t(\xi \vee \delta)$. Dually also $A t(\xi \vee \delta) \subseteq A t(\rho \vee \delta)$ and thus $\rho \vee \delta=\xi \vee \delta$.

Summarizing Lemmas $12-14$ we may formulate

THEOREM 4. If $\mathscr{C}(S)$ is atomistic then the relation $D$ is a complete congruence on $\mathscr{C}(S)$ and $\mathscr{C}(S) / D \cong \mathscr{C}(S / \delta) \cong \mathscr{C}$.

The lattices $[\delta, \omega]$ and $\mathscr{C}$ are two complete sublattices of $\mathscr{E}(S)$ and isomorphic copies of $\mathscr{C}(S) / D$. By Theorem 3 these lattices are isomorphic to $\mathscr{P}\left(X^{*}\right) \times \mathscr{C}(I)$ where $X=(S / \delta) / \mathscr{J}$ and $I$ is the kernel of $S / \delta$ if it 
exists and is the trivial semigroup otherwise. On the other hand, each $D$ class $\rho D$ has a greatest and a least element, $\rho^{D}$ and $\rho_{D}$, respectively. Then $[\delta, \omega]$ is precisely the set of all greatest elements $\rho^{D}$ whereas $\mathscr{C}$ is the set of all least elements $\rho_{D}$. By definition of $D$ and Lemma 14 it follows that $\rho^{D}=\rho \vee \delta$ and $\rho_{D}=\bigvee(A t(\rho) \cap \mathscr{B})$ for each $\rho \in \mathscr{C}(S)$. Furthermore, the $D$-class $\rho D=\left[\rho_{D}, \rho^{D}\right]$ coincides with the respective interval in the lattice of all equivalence relations on $S$.

THEOREM 5. For each $\rho \in \mathscr{C}(S), \rho D=\left\{\eta \in \mathscr{E}(S) \mid \rho_{D} \subseteq \eta \subseteq \rho^{D}\right\}$.

Proof. Let $\eta \in \mathscr{E}(S)$ be such that $\rho_{D} \subseteq \eta \subseteq \rho^{D}$ and $x \eta y$. Then $x \rho_{D} \vee \delta y$ implies $z x \rho_{D} z y$ and $x z \rho_{D} y z$. Therefore, $z x \eta z y$ and $x z \eta y z$ for all $z \in S$.

The greatest element $\varepsilon^{D}$ of $\varepsilon D$ is given by $\varepsilon^{D}=\delta$. For the least element of $\omega D$ we obtain the following explicit form.

PROPOSITION 4. $\omega_{D}=\bigvee \mathscr{B}=\omega_{S^{2}} \cup \varepsilon_{S}$.

Proof. We have $\omega_{D} \vee \delta=\omega^{D}=\omega$. For arbitrary $x, y \in S$ we have $x \omega_{D} \vee \delta y$ which implies that $z x \omega_{D} z y$ and $x z \omega_{D} y z$ for all $z \in S$. Hence $S / \omega_{D}$ is a null semigroup. By Result $2 S^{2}$ is globally idempotent. So we obtain that $\omega_{D} \mid S^{2}=\omega_{S^{2}}$. On the other hand, $\omega_{D}=\bigvee \mathscr{B}$. Let $x \in S \backslash S^{2}$ and $x \rho y$ for some atom $\rho$ and $x \neq y$. Now $\rho \mid S^{2}=\varepsilon_{S^{2}}$ and therefore $z x=z y$ and $x z=y z$ for all $z \in S$. In this case $\rho \subseteq \delta$. From this we conclude that

for all $\rho \in \mathscr{B}$. Hence

$$
\rho \mid\left(S \backslash S^{2} \cup\{x f\}\right)=\varepsilon_{S \backslash S^{2} \cup\{x f\}}
$$

$$
\omega_{D} \mid\left(S \backslash S^{2} \cup\{x f\}\right)=\varepsilon_{S \backslash S^{2} \cup\{x f\}}
$$

and we obtain that $\omega_{D}=\omega_{S^{2}} \cup \varepsilon_{S}$.

For semigroups $S$ characterized by Theorem 1 the relation $D$ simply is given by $\rho D \xi$ if and only if $\rho\left|S^{2}=\xi\right| S^{2}$. Furthermore, Theorem 3 implies that in this case $\mathscr{C}(S) \cong \mathscr{C}(S) / D \times \rho D$ for each $\rho \in \mathscr{C}(S)$.

Now let $\eta \in[\varepsilon, \delta]$ and $\mathscr{C}_{\eta}=\{\eta \vee \rho \mid \rho \in \mathscr{C}\}$. In the same way as in Lemma 14 it can be seen that $\bigvee G \subseteq \bigvee H$ if and only if $\eta \vee \vee G \subseteq \eta \vee \bigvee H$ for all $G, H \subseteq \mathscr{B}$. By the same reason as in Proposition 3 it follows that $\mathscr{C}_{\eta} \cong \mathscr{C}$. Also, $\mathscr{C}_{\eta}$ is a complete sublattice of $\mathscr{C}(S)$ with least element $\eta$ and greatest element $\eta \vee \omega_{S^{2}}$ and $\mathscr{C}_{\delta}=[\delta, \omega]$ and $\mathscr{C}_{\varepsilon}=\mathscr{C}$. 
We therefore have two interpretations of $\mathscr{C}(S)$ as a union of sublattices: on the one hand, $\mathscr{C}(S)$ is the disjoint union of the lattices $\left[\rho_{D}, \rho^{D}\right]$ which are intervals in the partition lattice of $S$. These intervals naturally are indexed by the elements of the atomistic congruence lattice $[\delta, \omega] \cong \mathscr{C}(S / \delta) \cong$ $\mathscr{P}\left(X_{S / \delta}^{*}\right) \times \mathscr{C}\left(I_{S / \delta}\right)$. On the other hand, $\mathscr{C}(S)$ is the union of the complete sublattices $\mathscr{C}_{\eta}$. Each of these lattices is isomorphic to the atomistic congruence lattice $\mathscr{P}\left(X_{S / \delta}^{*}\right) \times \mathscr{C}\left(I_{S / \delta}\right)$. These sublattices of $\mathscr{C}(S)$ naturally are indexed by the elements of $[\varepsilon, \delta]$ which is an interval in the partition lattice of $S$.

If $S^{2}$ is not weakly reductive then, since $\bigvee \mathscr{B}=\omega_{S^{2}} \cup \varepsilon_{S}$, the lattices $\mathscr{C}_{\eta}$ are not pairwise disjoint. If $S^{2}$ is weakly reductive then by Theorem 3 it follows that

$$
\mathscr{C}_{\eta}=\left\{\rho \in \mathscr{C}(S)|\eta|\left(S \backslash S^{2} \cup\{x f\}\right)=\rho \mid\left(S \backslash S^{2} \cup\{x f\}\right)\right\} .
$$

Therefore, in this (and only in this) case the lattices $\mathscr{C}_{\eta}$ are pairwise disjoint and the partition $\left\{\mathscr{C}_{\eta} \mid \eta \subseteq \delta\right\}$ induces a complete congruence on $\mathscr{C}(S)$.

\section{References}

[1] K. Auinger, 'Semigroups with complemented congruence lattices', Algebra Universalis 22 (1986), 192-204.

[2] K. Auinger, 'Weakly reductive semigroups with atomistic congruence lattices', J. Austral. Math. Soc. (Series A) 49 (1990), 59-71.

[3] L. M. Gluskin, 'Simple semigroups with zero', Dokl. Akad. Nauk. SSSR 103 (1955), 5-8 (Russian).

[4] E. J. Tully, Jr., 'Semigroups in which each ideal is a retract', J. Austral. Math. Soc. 9 (1969), 239-245.

Institut für Mathematik

Universität Wien

Strudlhofgasse 4

A-1090 Wien

Austria 